\title{
Short-term outcome of patients with closed comminuted femoral shaft fracture treated with locking intramedullary sign nail at Muhimbili Orthopaedic Institute in Tanzania
}

\author{
BILLY T. HAONGA ${ }^{1,2}$, FELIX S. MRITA 1 , EDMUND E. NDALAMA ${ }^{1}$ and JACKLINE E. MAKUPA ${ }^{3}$ \\ ${ }^{1}$ Muhimbili Orthopaedic Institute, P. O. Box 65474, Dar es Salaam, Tanzania \\ ${ }^{2}$ Muhimbili University of Health and Allied Sciences, Dar es Salaam, Tanzania \\ ${ }^{3}$ Ministry of Health and Social Welfare, P.O. Box 9083, Dar es Salaam Tanzania
}

\begin{abstract}
Background: Comminuted femoral shaft fractures are complex to treat because of increased risk of limb length discrepancies and mal-rotation deformities. Currently the interlocking intramedullary nail using image intensifiers is a suggested treatment for comminuted femoral shaft fractures. This study aimed to evaluate the short-term outcome of patients with closed comminuted femoral shaft fracture treated with locking intramedullary SIGN nail without image intensifiers at Muhimbili Orthopaedic Institute (MOI) in Tanzania.

Methods: A prospective hospital base study was conducted on patients with closed comminuted femoral shaft fractures admitted at MOI from March 2011 to February 2012. A total of 91 adult patients (18 to 84 years) were enrolled in the study. Of the total patients, 80 (88\%) patients were male, 11 were lost to followup, and 80 patients completed follow-ups of 18 weeks post operatively. Structured questionnaires and checklist forms were used to collect information. Operations were carried out either by antegrade or retrograde SIGN nail. Post operatively control radiographs, the lower limb length and mal-rotation deformities were assessed.

Results: Comminuted femoral shaft fracture accounted for $20.3 \%$ of all adult femoral shaft fractures admitted at MOI. Deep wound infection occurred in $2.5 \%$. Shortening of $2-3 \mathrm{~cm}$ was seen in $3.8 \%$ of the patients. No patient had shortening of more than $3 \mathrm{~cm}$. External rotation deformity of more than 20 degrees occurred in $6.3 \%$ of the cases. Internal rotation deformity of more than 15 degrees was seen in $7.9 \%$. Knee flexion of less than 90 degree was encountered in $7.5 \%$ of patients. By $18^{\text {th }}$ week $95 \%$ of the patients had callus formation.

Conclusion: SIGN nail appears to be an effective treatment for comminuted femoral shaft fractures in settings where image intensifiers and fracture tables are not readily available. They have excellent to good outcomes in rate of callus formation, limb length and limb alignment outcomes.
\end{abstract}

Keyword: femur, comminuted fracture, image intensifier, SIGN nail, Tanzania

Introduction

Comminuted femoral shaft fracture refers to fractures with multiple bone fragments and multiple fracture lines (Delahay \& Sauer, 2007). Femoral shaft fractures are among the most common fractures encountered in orthopaedic practice (Whittle \& Wood II, 2012). These fractures have age and gender-related bimodal distribution, with injuries occurring most frequently in young males following high-energy trauma. The second peak of injury is among elderly females following falls from standing due to postural instability and osteoporosis (Rockwood \& Green, 2006). Comminuted and segmental fractures are difficult to treat because of their vertical and rotational instability. The degree of fracture comminution is often classified using the Winquist-Hansen classification.

The type and location of the fracture, the degree of comminution and age of the patient may influence the method of treatment (Whittle \& Wood II, 2012). The treatment goals in patients with femoral shaft fracture includes: restoration of alignment rotation and length; preservation of the blood supply to aid union and prevent infection and rehabilitation of the extremity and the patient (Whittle \& Wood II, 2012)

Comminuted femoral shaft fractures can be treated operatively by open reduction and internal fixation (ORIF) using either plate or intramedullary (IM) nailing (Rockwood \& Green, 2006; Whittle \& Wood II, 2012;). Among these operative procedures; interlocking IM nailing is 
considered to be the treatment of choice for most femoral shaft fractures (Whittle \& Wood II, 2012). These fractures can be surgically managed by either open or closed nailing. Currently at MOI, Surgical Implant Generation Network (SIGN) nails are used in the fixation of femoral shaft fractures. The SIGN nails is solid interlocking intramedullary nail sub-type in which interlocking screws are applied with help of an external jig, and thus do not require image intensifiers (Zirkle, 2010).

Several studies have assessed the use of IM and its outcome. The average time to union has been reported to 13.5-13.8weeks (Johnson et al., 1984; Zuckerman 1987). The union rate of comminuted fractures treated with interlocking nail has been reported to range from $98.9 \%-99.2 \%$ in 4.4 months (Winquist et al., 1980; White et al., 1986). Fracture union in comminuted and rotationally unstable femur fractures treated with interlocking nails within 32 weeks has been reported in $98 \%$ of cases (Wiss et al., 1986). Other study reported that $97.6 \%$ of patients had union within a mean duration of 18.2 weeks (Tüzüner et al., 2002) and fracture consolidation has been found at a mean duration of 4.5 months in $86.5 \%$ of patients with severely comminuted fractures (Kempf et al., 1985). However, Borel et al. (1993) found that comminuted fractures unite within 23.25 weeks while Sulan et al. (2001) reported a mean time to union of 34 weeks without any additional surgical intervention.

The limb length discrepancy outcomes on comminuted femoral shaft fracture treated by interlocking IM nail has been reported by numerous authors. Shortening of more than $2 \mathrm{~cm}$ has been reported in $2.0 \%$ (Winquist et al., 1984) and 4.5\% (Arpacioglu et al., 2003) of patients. White et al. (1986) reported that 3.3\% patients had shortening of $1-2 \mathrm{~cm}$ in a study of 92 patients with comminuted femoral shaft fractures. However, shortening of $1-2 \mathrm{~cm}$ has been reported in a larger proportion (7.5\%) of patients by Sojbjerg et al. (1990). While Zuckerman et al. (1987) found that $3.2 \%$ of patients had shortening or lengthening of more than $1 \mathrm{~cm}$, limb length discrepancy of more than $1 \mathrm{~cm}$ and shortening of more than $2.5 \mathrm{~cm}$ have been observed in $14.3 \%$, and $2.3 \%$ of patients, respectively by Tüzüner et al. (2002). Limb length discrepancy of various lengths has been reported by other workers (Borel et al., 1993).

For the rotational deformity, several studies have reported an increased risk of limb malrotation outcomes among comminuted femoral shaft fracture patients treated with interlocking intramedullary nail. Mal-rotation deformity of more than 20 degrees has been reported in $1.7 \%$ $2.3 \%$ of patients (Winquist et al., 1980; Winguist et al., 1984). External rotation deformity of 5-10 degrees has been observed in 7.5\% of patients by Sojbjerg et al. (1990), while external rotation deformity of 10-35 degrees in $6 \%$ of patients has been reported by Borel et al. (1993). Furthermore, a study on 46 patients with comminuted fracture treated with interlocking IM nail, found that $2.2 \%$ of patients had an internal rotation of 10 degrees (Arpacioğlu et al., 2003).

According to a number of studies, infections have been rarely reported among patients with comminuted fractures (Wiss et al., 1980; Søjbjerg et al., 1990). However, infection rates of $0.4 \%-1.9 \%$ have been observed among patients with comminuted femoral fractures (Winquist \& Hansen, 1980; Winguist et al., 1984; Kempf et al., 1985). Flexion contractures of various degrees have also been reported among patients experiencing comminuted femoral fractures (Wiss et al., 1986; Sojbjerg et al., 1990; Nipatasaj et al., 1995).

Comminuted femoral shaft fractures are treated by using interlocking intramedullary nailing at MOI. Although anecdotal experience has been positive, the outcome of treatment has not been scientifically evaluated. This study was therefore carried out to evaluate the outcome of comminuted femoral shaft fractures treated by open reduction and fixation by interlocking SIGN nail.

\section{Materials and Methods}

\section{Study area and population}

Muhimbili Orthopaedic Institute (MOI) is located in Dar es Salaam, Tanzania. It is a tertiary hospital specialized in orthopaedics, traumatology and neurosurgery. It receives patients from all over the country and from neighbouring countries. It has a total bed capacity of 181 beds. The 
total inpatient admission for year 2010/2011 was 6,601 patients, with more than 4,500 operations. The inclusion criteria were patients aged 18 years or above, with closed comminuted femoral shaft fracture admitted at MOI. The exclusion criteria were patients with pathological fractures, bilateral femoral fractures, deformity of at least one lower limb (i.e. polio, major joint contractures, and amputees), patient with other underlying uncontrolled co-morbidity conditions (e.g. Diabetes mellitus, hypertension), Open comminuted fractures and patients who refused to participate were excluded to participate in the study.

\section{Study design, size and sampling technique}

Patients with comminuted femoral shaft fractures treated with interlocking intramedullary SIGN nails were followed at $2^{\text {nd }}, 6^{\text {th }}, 12^{\text {th }}$ and $18^{\text {th }}$ weeks post surgery to determine the short-term treatment outcome from March 2011 to February 2012. A total of 85 study subjects were recruited to the study and they were selected as they come until the required sample size was reached. Structured questionnaire were used to collect information, state of the wound, limb length, rotation deformity and range of motion of knee joint were assessed using a checklist forms. Other tools used were tape measure to measure true limb length, goniometer to measure limb rotation deformities and range of movement of knee joint.

\section{Examination, measurements and procedures}

Radiological examinations were conducted for both pre- and post- surgery. Pre-operative X-rays i.e. anteroposterior and lateral views of patient's affected femur were assessed and classified by the investigators and confirmed by a radiologist. The levels of femoral shaft fracture comminution were classified by using Winquist and Hansen classification (Winquist \& Hansen, 1980). An operative procedure was done by open reduction with interlocking IM SIGN nails were performed on all patients. Prophylactic antibiotics, typically $3^{\text {rd }}$ generation cephalosporins such as ceftriaxone were given during induction of anaesthesia. Antegrade nail was performed to those patients with proximal or middle third fractures, and retrograde nail was performed on distal third fractures and in obese patients. Patients were placed lateral decubitus or supine position on a standard operating table when antegrade or retrograde nail performed respectively. The reduction was performed under direct visualization with manual traction and manipulation. Image intensifier and fracture tables were not used. The nail was inserted through the tip of the greater trochanter for antegrade nails and through the intercondylar notch for retrograde nails. After insertion the SIGN nail was locked by 2, 3 or 4 interlocking screws based on individual surgeon decision. Cerclage wires were not used to stabilize the fracture fragments in any case.

Postoperative care and follow-ups, an immediate post-operative examination was done to assess the lower limb length and rotational deformities, and postoperative X-rays were obtained. Patients began physiotherapy programme before being discharged. Study subjects attended a follow-up clinic on the $2^{\text {nd }}$ week post operatively. They were assessed for the surgical wound, lower limb length and rotational alignment by physical examination. Additional followedup visits were scheduled at $6^{\text {th }}, 12^{\text {th }}$ and $18^{\text {th }}$ weeks postoperatively. During these visits anteroposterior and lateral femur X-rays were obtained. The union was assessed by number of cortices 0-4 bridged by callus (Whelan et al., 2002). Physical examination was also performed to assess range of knee motion, limb length, and mal-rotation deformity. Patients with reduced range of knee movements were scheduled to attend a regular physiotherapy. Those patients, who had no callus formation at the $18^{\text {th }}$ week, were considered to have delayed union, and appropriate treatments were given. Radiological union was defined as the presence of bridging callus across at least three cortices, and fractures were considered healed when the patients had radiological union, no tenderness to palpation and was able to bear weight without experiencing thigh pain. Thoresen scoring systems were used to classify mal-alignment and the range of knee motion with their degree outcome score range from excellent to poor (Thoresen et al., 1985). 
Data analysis

Statistical software (SPSS version 16) was used to analyse data. Continuous variables were summarized by mean $\&$ standard deviation and comparisons were made using student $\mathrm{t}$ - test. Categorical variables were summarized by frequency tables and comparisons were made using the Chi square or Fischer's exact test. The level of significance was set at $5 \%$.

\section{Ethical consideration}

This study received ethical approval from Muhimbili University of Health and Allied Science Ethics Committee. Only subjects who met the criteria and consented were selected to participate in the study.

\section{Results}

A total of 522 adult patients with femoral shaft fractures were admitted at MOI during the study period. Among these, 106 (20.3\%) patients had comminuted femoral shaft fracture. Of these, 91 patients met the study inclusion criteria. There were $88 \%$ (80/91) males and 12\% (11/91) females. The age ranged from 18 to 84 years with a mean age of $33 \pm 11.6$ years. The majority $(49.5 \%)$ of patients were $21-30$ years and $28.6 \%$ were $31-40$ years old. There were 11 patients (12\%) who were lost to follow up. Five patients were lost to follow up after discharge and six patients after the 6 week visit. The remaining 80 patients completed a minimum of 18 weeks of follow up after surgery.

Table 1: Time at first callus detection in relation to the number of interlocking screews used

\begin{tabular}{llllll}
$\begin{array}{l}\text { No. of interlocking } \\
\text { screws }\end{array}$ & No callus formation & $\mathbf{6}^{\text {th }}$ Week & $\mathbf{1 2}^{\text {th }}$ Week & $\mathbf{1 8}^{\text {th }}$ Week & Total \\
\hline 3 & $1(4.8 \%)$ & $8(38.0 \%)$ & $8(38.0 \%)$ & $4(19.0 \%)$ & 21 \\
4 & $3(5.1 \%)$ & $19(32.2 \%)$ & $20(33.9 \%)$ & $17(28.8 \%)$ & 59 \\
Total & $4(5.0 \%)$ & $27(33.8 \%)$ & $28(35.0 \%)$ & $21(26.2 \%)$ & 80 \\
\hline
\end{tabular}

Fischer exact test $\mathrm{P}$-value $=1.0$

According to Winquist and Hansen classification (Winquist \& Hansen, 1980), type II, III, IV and segmental fractures were encountered in $27.5 \%, 38.5 \%, 18.7 \%$ and $15.4 \%$ respectively. Forty eight patients $(59.3 \%)$ were operated within 24 hours of arrival and $24.3 \%$ within 48 hours from arrival due to various reasons including low Haemoglobin level and hemodynamically unstable patients. Clinically stable patients (30.8\%) were discharged 24 hours posts operatively. Four patients (5\%) developed post-operative surgical site infection; 2 patients $(2.5 \%)$ had deep infections and 2 (2.5\%) superficial infections. Fifty-five patients $(68.8 \%)$ had callus formation by $12^{\text {th }}$ week. The mean duration to callus formation was $10.9 \pm 5.3$ weeks. Ninety five percent of patients $(n=80)$ had callus formation by $18^{\text {th }}$ week post-surgery. Only four ( $5 \%$ ) patients had no callus by $18^{\text {th }}$ week. There was no statistical significance difference in time to callus formation between patients treated with 3 or 4 interlocking screws ( $p$ - value $=1.0$ ) (Table 1$)$.

Table.2. Limb length at $18^{\text {th }}$ week outcome against number of interlocking screw used

\begin{tabular}{lllll}
\hline No. screws & Normal & Shortening $<\mathbf{2 c m}$ & Shortening $\mathbf{2 - 3} \mathbf{~ c m}$ & Total \\
\hline 3 & $17(81.0 \%)$ & $4(19.0 \%)$ & $0(0.0 \%)$ & 21 \\
4 & $47(79.7 \%)$ & $9(15.3 \%)$ & $3(5.0 \%)$ & 59 \\
Total & $64(80.0 \%)$ & $13(16.2 \%)$ & $3(3.8 \%)$ & 80 \\
\hline
\end{tabular}

Fischer exact test $p$ value $=1.0$

\section{Limb length discrepancy}

Limb shortening of 2 to $3 \mathrm{~cm}$ occurred in $3.8 \%$ of patients. No patients had shortening of more than $3 \mathrm{~cm}$. According to Thoresen criteria $96.2 \%$ of patients had excellent to good limb length outcome. There was no statistically significant difference in limb length outcome between patients treated with 3 or 4 interlocking screws $(p$-value $=1.0)($ Table 2$)$. 
Tanzania Journal of Health Research

Volume 17, Number 3, July 2015

Doi: http://dx.doi.org/10.4314/thrb.v17i3.8

\section{Rotational deformity}

External rotation deformity of more than 20 degrees was observed in $6.3 \%$ of patients. Internal rotation deformity of more than 15 degrees occurred in $8.9 \%$. According to Thoresen criteria $76.3 \%$ of patients had excellent limb alignment outcome (Table 3).

Table 3: The distribution of limb alignment outcome at $18^{\text {th }}$ week post- surgery

\begin{tabular}{lllll}
\hline Limb alignment & Degree & Frequency & Percent & Thoresen score \\
\hline Normal & & 51 & 63.8 & Excellent \\
External rotation deformity & $11-15$ & 10 & 12.5 & Good \\
& $16-20$ & 3 & 3.8 & Fair \\
& $21-25$ & 2 & 2.5 & Poor \\
Internal rotation deformity & $>25$ & 3 & 3.8 & Poor \\
& $11-15$ & 4 & 5.0 & Fair \\
& $16-20$ & 1 & 1.3 & Poor \\
& $21-25$ & 3 & 3.8 & Poor \\
& $>25$ & 3 & 3.8 & Poor \\
& Total & 80 & 100.0 & \\
\hline
\end{tabular}

\section{Range of movement of knee joint}

Overall knee flexion of less than 90 degree was observed in $7.5 \%$ of the patients. Knee flexion of less than 90 degrees was seen in $3.3 \%$ of patients treated with antegrade and it occurred in $20 \%$ patients treated by retrograde nail. The difference was statistically significant ( $p$-value $=0.032$ ). By Thoresen criteria excellent to good knee flexion deficit was encountered in $87.5 \%$ of patients (Table 4).

Table 4: Distribution of ranges of degree of knee flexion of affected limb

\begin{tabular}{|c|c|c|c|c|c|}
\hline Treatment & $<90^{\circ}$ & $91-110^{\circ}$ & $111-120^{\circ}$ & $>120^{\circ}$ & Total \\
\hline Antegrade & $2(3.3 \%)$ & $4(6.7 \%)$ & $1(1.6 \%)$ & $53(88.3 \%)$ & 60 \\
\hline Retrograde & $4(20.0)$ & $0(0.0 \%)$ & $1(5.0 \%)$ & $15(75.0 \%)$ & 20 \\
\hline Total & $6(7.5 \%)$ & $4(5.0 \%)$ & $2(2.5 \%)$ & $68(85.0 \%)$ & 80 \\
\hline
\end{tabular}

Fisher exact test $p$-value $=0.032$

\section{Discussion}

This study found that, $95 \%$ of the patients had callus formation by the $18^{\text {th }}$ week postoperatively, similar to findings by other workers (Wiss et al., 1986; White et al., 1986; Webb et al., 1988). However, other workers have reported shorter periods (13.5-16.5 weeks) of callus formation (Johnson et al., 1984; Zukerman et al., 1987; Arpiciglu et al., 2003). These differences may be due to variability between studies in the definition of fracture union or could be due to different mode of reduction (open versus closed).

By Thoresen criteria score, majority of the patients in our study had excellent or good limb length outcome. Our findings are comparable to those reported by others (Winquist \& Hansen, 1980; Arpacioğlu et al., 2003). Shortening of $1-2 \mathrm{~cm}$ in this study was seen in $16.2 \%$ of patients and was better than a shortening reported by Kempf et al. (1985). However these results were higher those reported previously by other workers (Wiss et al., 1986; White et al., 1986; Zuckerman et al., 1987). The difference is likely to be due to the fact that in this study length was maintained by manual traction, which might cause loss of length while in other studies patients affected limb length were compared with normal limbs and maintained by traction table and the length of nail was estimated from radiograph of normal contralateral femora.

About two thirds of patients had normal limb alignment. External rotation deformity in comminuted fractures is a common complication. Our findings revealed that external and internal rotation of more than 15 degrees occurred in less than $10 \%$ of the patients. According to Thoresen criteria score over three-quarters of our patients had excellent limb alignment outcome. Similar results have been observed by Wiss et al. (1986) and Borel et al. (1993). The 

similar to findings by Sojbjerg et al. (1990). However, in a previous study Wissa et al. (1986) had reported contrary results. In a study in Thailand, Nipatasaj et al. (1995) reported that, all patients had more than 9o-degree knee flexion at 2 weeks post operatively. The difference may be due to lack of regular physiotherapy in our setting, or due to severe injury which delayed the initiation of knee flexion exercise.

In this study, only two patients had deep wound infection similar observations by Kempf et al. (1985). However, the proportion of patients with infection in our study was higher than those reported by others elsewhere (Winquist \& Hansen, 1980; Winguist et al., 1984). The observed difference might be due to the high rate of open reduction in this study, which is necessary in the absence of fluoroscopic imaging.

Our study had a number of limitations. There was no randomization of treatment, such as the number of screws, which can lead to confounding bias; the follow up was limited to 18 weeks, which is not adequate to fully assess the rate of radiographic union or the rate of deep infection; and there was lack of a standardized method for assessing radiographic union, limits the ability to determine union.

In conclusion, the comminuted femoral shaft fracture treated with interlocking intramedullary SIGN nail has excellent to good outcome in rate of callus formation, limb length and limb alignment outcomes. SIGN nail appears to be an effective treatment for comminuted femoral shaft fractures in settings where image intensifiers and fracture tables are not readily available.

\section{References}

Arpacioğlu, M.O., Akmaz, I., Mahiroğlullar, M., Kiral, A. \& Rodop, O. (2003) Treatment of femoral shaft fractures by interlocking intramedullary nailing in adults. Acta Orthopeadica Traumatology Turkey 37, 203-212.

Borel, J.C., Dujardin, F., Thomine, J.M. \& Biga, N. (1993) Closed locked nailing of complex femoral fractures in adults:[ a propos of 68 cases]. Revue de Chirurgie Orthopédique et Réparatrice de l'appareil Moteur 79, 553-564.

Delahay, J.N. \& Sauer, S.T. (2007) Essentials of Orthopeadic Surgery. Third edition, Springer, Washington, DC.

Johnson, K.D., Johnston, D.C., \& Parker, B. (1984) Communited femoral-shaft fractures: treated by roller traction, cerclage wires and an intermedullary nail, or interlocking intermedullary nail. Journal of Bone and Joint Surgery (Am) 66A, 1222-1235.

Kempf, I., Grosse, A. \& Beck, G. (1985) Closed locked intramedullary nailling: its application to comminuted fractures of the femur. Journal of Bone and Joint Surgery (Am) 67, 709-720.

Nipatasaj, P., Bhovati, V., Patanakumjorn, D. \& Jirarachvaro, S. (1995) Closed Intramedullary nailing for femoral shaft fracture in Ratchaburi Hospital. Journal of Medical Association of Thailand 78, 419-423.

Ostrum, R.F., Verghese, G.B. \& Santner, T.J. (1993) The lack of association between femoral shaft fractures and hypotensive shock. Orthopaedic Trauma 7, 338-342.

Rockwood, C.A. \& Green, D.P. (2006) Fractures in Adults. Philadelphia: Lippincott Williams \& Wilkins.

Sojbjerg, J.O., Eiskjaer, S. \& Moller-larsen, F. (1990) Locked nailing of comminuted and unstable fractures of the femur. Journal of Bone and Joint Surgery $(\mathrm{Br}), 72,23-25$.

Sultan, S. (2001) Interlocked nailing of comminuted fractures shaft of femur. Journal of Ayoub Medical College Abottabad 13, 14-15.

Thoresen, B.O., Alho, E.A. \& Stromsoe, K. (1985) Interlocking intramedullary nailling in femoral shaft fractures.A report of 48 cases. Journal of Bone and Joint Surgery (Am) 67, 313-1320.

Tüzüner, T., Subasi, M., Kapukaya, A. \& Necmioglu, N.S. (2002) Treatment of femoral shaft fractures with interlocking intramedullary nailing. Acta Orthopeadica Traumatology Turkey 36, 211-219. 
Webb, L.X., Anthony, G. \& Fowler, H.L. (1988) Unstable Femoral shaft fracture: A comparison of interlocking nailing versus traction and casting methods. Journal of Orthopeadic Trauma 2, 10-12.

Whelan, M.B., Mckee, M.D. \& Guyat, G.H. (2002) Interrobserver and intraobserver variation in assessment of healing of tibia after intramedullary fixation. Journal of Bone and Joint Surgery (BR) 84-B, 15-18.

White, G.M., Healy, W.L., Brumback, R.J., Burgess, A.R. \& Brooker, A.F. (1986) The treatment of fractures of the femoral shaft with the Brooker- wills distal locking intramedullary nail. Journal of Bone and Joint Surgery (Am) 68, 865-876.

Whittle, P. \& Wood II, G.W. (2012) Fractures of shaft of femur. In: Campbell's Operative Orthopaedics. $11^{\text {th }}$ Edition fractures of shaft of femur. Philadelphia: Elsevier Mosby.

Winguist, R.A., Hansen, S.T. \& Clawson, D.K. (1984) Closed intramedullary nailing of femoral fractures $>$ A report of five hundred and twenty cases. Journal of Bone and Joint Surgery (Am) 66A, 529-539.

Winquist, R.A. \& Hansen, S.T. (1980) Comminuted fractures of the femoral shaft treated by intramedullary nailing. Orthopedic Clinics of North America 11, 633-648.

Wiss, D.A., Fleming, C.H., Matta, J.M. \& Clark, D. (1986) Comminuted and rotationally unstable fractures of the femur treated with an interlocking nail. Clinical Orthopeadics \& Related Research 212, 35-47.

Zirkle, L.G. (2010) Techinique Manual of SIGN IM and Interlocking Screw System Insection and Extraction Guide. http://signsurgeons.org/wp-content/uploads/2012/07/TM-20120604.pdf

Zuckerman, J.D., Veith, R.G., Johnson, K.D. \& Bach, A.W. (1987) Treatment of ustable femoral shaft fractures with closed interlocking intramedullary nailing. Journal of Orthopeadic \& Trauma 1, 209-218. 